Ambiente \& Água - An Interdisciplinary Journal of Applied Science
ISSN 1980-993X - doi:10.4136/1980-993X
www.ambi-agua.net
E-mail: ambi.agua@gmail.com

\title{
Variabilidade espacial do estoque de carbono e atributos físicos do solo em terra preta arqueológica sob pastagem
}

\author{
ARTICLES doi:10.4136/ambi-agua.2002
}

Received: 11 Sep. 2016; Accepted: 10 Jul. 2018

\begin{abstract}
Marcelo Dayron Rodrigues Soares ${ }^{1}$; Milton César Costa Campos ${ }^{1 *}$; José Maurício da Cunha'; Zigomar Menezes de Souza ${ }^{2}$; Ivanildo Amorim de Oliveira ${ }^{3}$; Renato Eleotério de Aquino ${ }^{3}$; Bruno Campos Mantovanelli ${ }^{4}$, Ludimila Souza Oliveira ${ }^{1}$

\author{
${ }^{1}$ Universidade Federal do Amazonas (UFAM), Humaitá, AM, Brasil \\ Instituto de Educação, Agricultura e Ambiente (IEAA). E-mail: marcelo.dayron@gmail.com, \\ mcesarsolos@gmail.com,maujmc@gmail.com, ludimila.ufam@outlook.com \\ ${ }^{2}$ Universidade Estadual de Campinas (UNICAMP), Campinas, SP, Brasil \\ Faculdade de Engenharia Agrícola. E-mail: zigomams@agri.unicamp.br \\ ${ }^{3}$ Universidade Estadual Paulista "Júlio de Mesquita Filho" (UNESP), Jaboticabal, SP, Brasil \\ Programa de Pós-Graduação em Agronomia. E-mail: ivanildoufam@gmail.com, aquino.rea@gmail.com \\ ${ }^{4}$ Universidade Federal Rural de Pernambuco (UFRPE), Recife, PE, Brasil \\ Departamento de Agronomia (DEPA). E-mail: brunomantovanelli21@gmail.com \\ "Corresponding author
}

\section{RESUMO}

Algumas áreas de Terra Preta Arqueológica na Amazônia vêm sendo utilizadas com pastagem o que tem gerado grande preocupação quanto às alterações dos atributos físicos e o estado de agregação do solo. O objetivo deste estudo foi avaliar a variabilidade espacial e as possíveis modificações dos atributos físicos e agregados do solo em terra preta arqueológica sob pastagem. Uma grade de $80 \times 56 \mathrm{~m}$ foi usada e a amostragem realizada em 88 pontos em disposição de 8 x 8m. Nas camadas de $0-0,05,0,05-0,10$ e $0,10-0,20 \mathrm{~m}$ foram avaliados: a densidade do solo (Ds), carbono orgânico total (COT), estoque de carbono (Est C), diâmetro médio ponderado (DMP), macroporosidade (Macro), microporosidade (Micro) e volume total de poros (VTP). Pelo exame de semivariogramas constatou-se a ocorrência de dependência espacial. Mesmo a área de estudo sendo em pastagem observou-se que os valores de Ds, Macro e VTP, estiveram acima ou abaixo dos valores de referências que podem causar restrição ao crescimento radicular de plantas e à infiltração de água no solo. O DMP e Ds foram dependentes do COT, pois os valores de ambos aumentam ou diminuem de acordo com a camada. Com o aumento da camada do solo, houve o acréscimo do estoque de carbono.

Palavras-chave: estrutura do solo, geoestatística, manejo.

\section{Spatial variability of physical attributes and soil aggregates in archaeological dark dirt under pasture}

\begin{abstract}
Some areas of archaeological dark dirt in the Amazon have been used with pasture that has generated great concern regarding changes in soil physical attributes and its aggregation state. The objective of this study was to evaluate the spatial variability and possible modifications of soil physical and aggregate attributes in archaeological black dirt under pasture. A grid of $80 \mathrm{x}$
\end{abstract}


$56 \mathrm{~m}$ with 88 sampling points distributed in $8 \times 8 \mathrm{~m}$ were marked. Soil layers at 0-0.05, 0.050.10 , and $0.10-0.20 \mathrm{~m}$ were analyzed for: soil density (SD), total organic carbon (TOC), carbon stock (C stock), meanweight diameter (MWD), macroporosity (Macro), microporosity (Micro), and total porosity (TP). From semivariograms tests it was verified the occurrence of spatial dependence. Even with major pasture cover in the study area it was observed that the values of Ds, Macro, and VTP were above or below the reference values, which may cause restriction for root growth of plants and water infiltration in the soil. The DMP and Ds were COT-dependent because values of both increase or decrease depending on the soil layer. When higher the soil layer there was an increase in carbon stock.

Keywords: geostatistics, management, soil structure.

\section{INTRODUÇÃO}

A diversidade geológica existente na Amazônia, aliada ao relevo diferenciado e sob a influência de fortes temperaturas e precipitações, resultaram na formação das mais variadas classes de solo. Uma das principais características dos solos da Amazônia é sua baixa fertilidade, no entanto, os solos denominados de terras pretas arqueológicas (TPAs) ocorrentes nessa região têm se destacado por sua elevada fertilidade natural.

As terras pretas arqueológicas são solos com características de coloração escura, com presença de fragmentos cerâmicos e/ou líticos incorporados aos seus horizontes mais superficiais (Kämpf e Kern, 2005) e apresentam teor alto a muito alto de fósforo disponível (Campos et al., 2011). Esses solos ocorrem em manchas descontínuas por toda a Amazônia e tem como destinação principal o plantio de subsistência pelas populações locais. Devido ao pouco conhecimento do potencial agrícola, essas áreas vêm sendo convertidas de áreas de pastagens, principalmente na região sul do Amazonas, por sistemas extrativistas rudimentares que promovem modificações nos atributos físicos do solo.

Dentre os atributos físicos mais sensíveis a essas mudanças, destacam-se: a estabilidade de agregados, densidade do solo, a macroporosidade e teor de carbono orgânico. Tais atributos são indicadores utilizados para verificar as alterações no solo (Vasconcelos et al., 2010; Vieira et al., 2011). Dentre as diversas maneiras de verificar mudanças nos atributos do solo, a análise geoestatística é uma das mais eficazes (Alho et al., 2014). O comportamento dos atributos físicos pode ser monitorado pela distribuição espacial. Segundo Souza et al. (2004), os atributos físicos variam entre pontos relativamente próximos em área de mesma unidade taxonômica, muitas vezes de forma significativa.

Dado à necessidade de entender a variabilidade espacial dos atributos físicos e o carbono orgânico do solo, vários estudos já foram realizados enfatizando variações causadas pelo próprio uso e manejo em áreas de TPAs (Aquino et al., 2014b; Oliveira et al., 2013), porém o conhecimento ainda é escasso e não há informações que tratam a variabilidade espacial dessas áreas sob pastagem.

Dessa forma, o objetivo desse trabalho foi verificar a variabilidade espacial dos atributos físicos e o estado de agregação do solo em área de terra preta arqueológica sob pastagem na região de Manicoré, AM.

\section{MATERIAL E MÉTODOS}

A área de estudo está situada no sul do Estado do Amazonas, nas mediações da comunidade de Santo Antônio do Matupi, às margens da BR 230, Transamazônica, na região de Manicoré, AM. As coordenadas geográficas são $07^{\circ} 53^{\prime} 36,84^{\prime \prime}$ de latitude Sul e $61^{\circ} 23^{\prime} 54,49^{\prime \prime}$ de longitude Oeste e com altitude média de $83 \mathrm{~m}$. De acordo com a classificação de Köppen, o clima da 
região é do tipo tropical chuvoso, apresentando um período seco de pequena duração (Am), temperaturas variando entre 25 e $27^{\circ} \mathrm{C}$ e precipitação média varia entre de 2.500 e $2.750 \mathrm{~mm}$ ao ano (Brasil, 1978).

O material de origem é proveniente da Formação Içá, caracterizada com produto de sedimentação pleistocênica, coberta por depósitos eólicos. O relevo é constituído por platôs com superfícies planas, sendo a zona de borda marcada por colinas e cristas alinhadas e localmente escarpadas (CPRM, 2001). A pastagem foi implantada há sete anos sob pastejo extensivo com o cultivo de Brachiaria brizanta. A identificação dos horizontes, a descrição morfológica e coleta de amostras dos horizontes dos solos foram realizadas conforme Dos Santos et al. (2013) e o solo foi classificado como Argissolo Vermelho Amarelo Distrofico típico segundo critérios estabelecidos pelo SiBCS (EMBRAPA, 2013).

Estabeleceu-se uma malha amostral de $80 \times 56 \mathrm{~m}$ que continham 88 pontos de amostragem georreferenciados dispostos em malha de $8 \times 8 \mathrm{~m}$. Em cada ponto realizou-se a coleta de solo para as amostras deformadas e indeformadas nas camadas: 0-0,05; 0,05-0,010 e 0,10-0,20m (Figura 1).
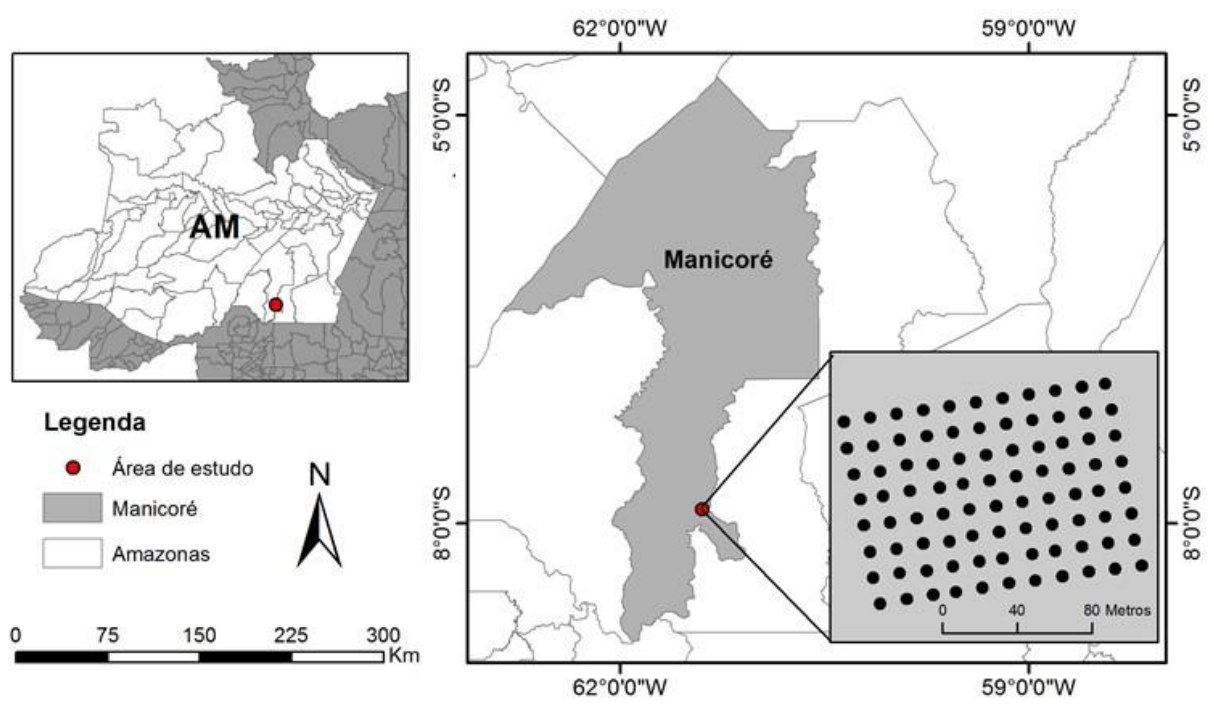

Figura 1. Mapa de localização da área de estudo e malha amostral.

O estado de agregação das amostras de solo foi avaliado pelo índice de diâmetro médio ponderado (DMP), por via úmida, conforme metodologia descrita por Kemper e Chepil (1965). As amostras de solos foram retiradas em forma de blocos, as quais foram secas ao ar e passadas em peneiras de $9,52 \mathrm{~mm}$ e $4,76 \mathrm{~mm}$. Em seguida, os agregados retidos na peneira de $4,76 \mathrm{~mm}$ foram empregados na análise da estabilidade de agregados via úmida, colocadas sobre um jogo de peneiras com tamisagem de $2,0 \mathrm{~mm} ; 1,0 \mathrm{~mm} ; 0,5 \mathrm{~mm} ; 0,25 \mathrm{~mm} ; 0,105 \mathrm{~mm}$; e $0,063 \mathrm{~mm}$ e submetendo-os a oscilações verticais. Transcorridos 15 minutos, as porções retidas em cada peneira foram transferidas para potes de alumínio com auxílio de jatos de água e secas em estuda a $105^{\circ} \mathrm{C}$ por um período de $24 \mathrm{~h}$ para posterior pesagem.

O carbono orgânico total (COT) foi determinado pelo método de Walkley-Black modificado por Yeomans e Bremner (1988). O estoque de carbono (Est C) foi determinado pela expressão proposta por Veldkamp (1994): Est $\mathrm{C}=(\mathrm{CO} \times \mathrm{Ds} \times \mathrm{e}) / 10$, em que Est $\mathrm{C}=$ estoque de carbono orgânico $\left(\mathrm{Mg} \mathrm{ha}^{-1}\right)$; COT = teor de carbono orgânico total $\left(\mathrm{g} \mathrm{kg}^{-1}\right)$; Ds = densidade do solo $\left(\mathrm{kg} \mathrm{dm}^{-3}\right)$; e = espessura da camada $(\mathrm{cm})$.

As amostras de solo indeformadas foram saturadas por meio da elevação gradual de uma lâmina de água até atingir cerca de $2 / 3$ da altura do anel. O volume total de poros (VTP) foi obtida pela diferença entre a massa do solo saturado e a massa do solo seco em estufa a $105^{\circ} \mathrm{C}$ durante $24 \mathrm{~h}$. A microporosidade (Micro) do solo foi determinada pelo método da mesa de

\section{IPABH}

Rev. Ambient. Água vol. 13 n. 6, e2002 - Taubaté 2018 
tensão a 6 kPa, conforme Embrapa (1997). Pela diferença entre a VTP e a Micro, obteve-se a macroporosidade (Macro). A densidade do solo (Ds) foi calculada pela relação entre a massa seca a $105^{\circ} \mathrm{C}$ durante $24 \mathrm{~h}$ da amostra de solo do cilindro volumétrico e o volume do mesmo.

Para caracterização da variabilidade espacial, foi utilizada a análise geoestatística. $\mathrm{O}$ semivariograma experimental foi estimado pela Equação 1.

$$
\hat{\gamma}(h)=\frac{1}{2 N(h)} \sum_{i=1}^{N(h)}\left[Z\left(x_{i}\right)-Z\left(x_{i}+h\right)\right]^{2}
$$

em que:

$\gamma(\mathrm{h})$ - semivariância estimada a partir dos dados experimentais;

$\mathrm{Z}$ - valores medidos nos pontos xi e xi $+\mathrm{h}$;

$\mathrm{N}(\mathrm{h})$ - número de pares de valores medidos separados por uma distância h;

Com os dados obtidos foram efetuadas a estatística descritiva dos dados e a hipótese de normalidade avaliada pelo teste de Kolmogorov-Smirnov por meio do software Minitab 14. Os semivariogramas foram ajustados ao melhor modelo matemático por meio da validação cruzada (VC) "cross-validation" e coeficiente de terminação $\left(\mathrm{R}^{2}\right)$ pelo software GS+. Para a confecção dos mapas espaciais utilizou-se o software Surfer versão 8.0.

O grau de variabilidade foi estimado pelo coeficiente de variação (CV) dos atributos, conforme sugerido por Warrick e Nielsen (1980) que consideram baixa variabilidade quando $\mathrm{CV}<12 \%$; média para o intervalo $12-60 \%$ e alta variabilidade quando CV $>60 \%$. A análise do grau de dependência espacial (GDE) foi realizada segundo Cambardella et al. (1994) que preconizam a proporção em porcentagem do efeito pepita $\left(\mathrm{C}_{0}\right)$ em relação ao patamar $\left(\mathrm{C}_{0}+\mathrm{C}_{1}\right)$ e apresentam: (a) dependência forte <25\%; (b) dependência moderada entre 25 e $75 \%$ e (c) dependência fraca $>75 \%$.

\section{RESULTADOS E DISCUSSÃO}

Os resultados da análise descritiva dos dados indicaram ajuste a uma distribuição normal (Tabela 1). Os índices de assimetria e curtose foram próximos de zero, com exceção do DMP e Micro na camada $0,05-0,10 \mathrm{~m}$. A média e a mediana para todos dos atributos foram próximos. De acordo com Campos et al. (2013), quando uma distribuição é simétrica, os valores da média e mediana são coincidentes. Em todos os casos, com exceção para o DMP, os atributos ajustaram-se à distribuição normal determinada por meio do teste de Kolmogorov-Smirnov.

Os valores médios de densidade do solo variaram entre 1,27 a $1,29 \mathrm{~kg} \mathrm{~m}^{-3}$, estes resultados estão de acordo com os obtidos por Dos Santos et al. (2013) em estudos com caracterização de TPA's no Sul do Amazonas. Segundo Steinbeiss et al. (2009), a baixa Ds está relaciona aos altos teores de carbono orgânico e a intensa atividade biológica. Mesmo sob pastagem, as menores Ds são típicos em áreas de TPA. De modo geral, esses resultados estão abaixo do valor crítico para Ds, conforme Blaiski et al. (2008), os quais afirmam que valores de Ds acima de $1,5 \mathrm{~kg} \mathrm{~m}^{-3}$ promovem significativas restrições ao desenvolvimento radicular das plantas.

Quanto a agregação do solo, observou-se que os agregados estáveis e de maior diâmetro (DMP) foram encontrados de forma contínua nas camadas de 0,0-0,05 e 0,05-0,10m. Os menores valores foram verificados na camada $0,10-0,20 \mathrm{~m}$. Em estudos realizados com variação espacial dos agregados em terra preta arqueológica, Rocha Silva et al. (2011) observaram que a variação do DMP por camada é reduzida à medida que aumenta a camada avaliada. Para Campos et al. (2012), como esses solos são de origem antrópica, a alta concentração de matéria orgânica derivada da queima parcial do carvão vegetal pode deixar este solo mais solto e, consequentemente, com a predominância de formação de microagregados. Rozane et al. (2010), 
avaliando os agregados em diversos manejos, verificaram os maiores valores de DMP na camada superficial de área de pastagem.

Tabela 1. Medidas descritivas dos dados do diâmetro médio ponderado (DMP), densidade do solo (Ds), carbono orgânico total (COT), macroporosidade (Macro), microporosidade (Micro), volume total de poros (VTP) em diferentes camadas.

\begin{tabular}{|c|c|c|c|c|c|c|c|}
\hline & DMP & Ds & Est C & COT & Macro & Micro & VTP \\
\hline & $(\mathrm{mm})$ & $\mathrm{kg} \mathrm{cm}^{-3}$ & Mg há ${ }^{-1}$ & $\mathrm{~g} \mathrm{~kg}^{-1}$ & & $\mathrm{~m} \mathrm{~m}^{-3}$ & \\
\hline & \multicolumn{7}{|c|}{$0,00-0,05 \mathrm{~m}$} \\
\hline Média & 3,14 & 1,29 & 88,92 & 137,06 & 0,11 & 0,24 & 0,39 \\
\hline Mediana & 3,16 & 1,30 & 89,05 & 136,93 & 0,12 & 0,23 & 0,38 \\
\hline $\mathrm{CV}^{1}$ & 3,14 & 9,13 & 8,75 & 4,06 & 23,47 & 11,74 & 8,81 \\
\hline Curtose & 0,71 & $-0,01$ & $-0,10$ & 1,49 & 0,55 & 2,98 & 1,52 \\
\hline Assimetria & 1,03 & 0,37 & $-0,33$ & $-0,72$ & 0,47 & 0,05 & 0,37 \\
\hline \multirow[t]{2}{*}{$\mathrm{d}^{2}$} & $0,03^{\mathrm{ns}}$ & $0,15^{*}$ & $0,15^{*}$ & $0,15^{*}$ & $0,08^{*}$ & $0,08^{*}$ & $0,09^{\prime \prime}$ \\
\hline & \multicolumn{7}{|c|}{$0,05-0,10 \mathrm{~m}$} \\
\hline Média & 3,12 & 1,27 & 86,16 & 135,28 & 0,18 & 0,24 & 0,42 \\
\hline Mediana & 3,19 & 1,29 & 86,75 & 135,56 & 0,17 & 0,23 & 0,40 \\
\hline $\mathrm{CV}^{1}$ & 5,52 & 8,05 & 7,22 & 1,68 & 21,43 & 11,08 & 9,09 \\
\hline Curtose & 7,74 & $-0,74$ & $-0,67$ & 0,41 & 1,07 & 4,78 & 0,59 \\
\hline Assimetria & $-2,49$ & 0,17 & $-0,15$ & $-0,68$ & 0,57 & 1,18 & 0,10 \\
\hline \multirow[t]{2}{*}{$\mathrm{d}^{2}$} & $0,01^{\mathrm{ns}}$ & $0,15^{*}$ & $0,15^{*}$ & $0,15^{*}$ & $0,15^{*}$ & $0,15^{*}$ & $0,15^{*}$ \\
\hline & \multicolumn{7}{|c|}{$0,10-0,20 \mathrm{~m}$} \\
\hline Média & 3,01 & 1,27 & 164,19 & 133,91 & 0,22 & 0,21 & 0,43 \\
\hline Mediana & 3,07 & 1,23 & 163,9 & 134,1 & 0,23 & 0,21 & 0,44 \\
\hline $\mathrm{CV}^{1}$ & 6,55 & 8,39 & 7,69 & 1,56 & 15,07 & 11,84 & 7,67 \\
\hline Curtose & 0,59 & 0,10 & 0,20 & 1,18 & 0,03 & 1,26 & $-0,16$ \\
\hline Assimetria & $-1,01$ & 0,22 & 0,16 & 0,50 & 0,07 & 0,69 & 0,17 \\
\hline $\mathrm{d}^{2}$ & $0,01^{\mathrm{ns}}$ & $0,15^{*}$ & $0,15^{*}$ & $0,15^{*}$ & $0,15^{*}$ & $0,09^{*}$ & $0,15^{*}$ \\
\hline
\end{tabular}

${ }^{1} \mathrm{CV}$ : coeficiente de variação; ${ }^{2} \mathrm{~d}$ : teste de normalidade Kolmogorov-Smirnov, *significativo a $5 \%$ de probabilidade.

Menores valores do estoque de carbono foram obtidos nas duas camadas superficiais, o que foi influenciado, principalmente, pelos maiores valores de densidade do solo. Provavelmente a maior densidade do solo na camada superficial é decorrente do pisoteio do gado. O maior valor do estoque de carbono foi encontrado na última camada. Em estudos com alguns atributos físicos em diversas áreas, entre elas, pastagem, Coutinho et al. (2017) afirmam que há influência do carbono orgânico do solo sobre a Ds, uma vez que onde foram obtidos os maiores valores de carbono orgânico foi onde se quantificou os menores valores de Ds e viceversa.

Os coeficientes de variação $(\mathrm{CV})$ indicaram homogeneidade dos dados $(\mathrm{CV}<12)$ segundo Warrick e Nielsen (1980). Como exceção, a macroporosidade teve valores de CV medianos $(23,47 \%, 21,4 \%$ e $15,07 \%$ respectivamente) em concordância com os resultados obtidos por Aquino et al. (2014b) em estudo com área de pastagem e Aquino et al. (2014a) em área com TPA.

Constatou-se que os valores médios da Micro permaneceram praticamente constantes nas duas primeiras camadas, com pequena diminuição na última camada. Já a Macro e a porosidade total mostraram comportamento inverso a Micro, com isso, o aumento da Ds pode reduzir a

\section{IPABH}

Rev. Ambient. Água vol. 13 n. 6, e2002 - Taubaté 2018 
macroporosidade e elevar a Micro. Para Santos et al. (2012), o crescimento das raízes pode ser prejudicado quando a macroporosidade do solo é reduzida a valor inferior a $0,15 \mathrm{~m}^{3} \mathrm{~m}^{-3}$. No entanto, os resultados ficaram acima do valor de referência.

Os parâmetros dos modelos ajustados aos semivariogramas para os diferentes atributos são apresentados na Tabela 2. Os valores da validação cruzada (VC) oscilaram entre 0,61 e 1,01. De acordo com Alho et al. (2014) quanto mais próximos de 1 a VC maior a confiabilidade e mais eficiente o modelo para representar o estudo. O coeficiente de determinação $\left(r^{2}\right)$ dos semivariogramas revelam ótimos ajustes, oscilando entre 0,52 e 0,97. Todavia, é válido considerar que os atributos tiveram os menores valores de $\mathrm{C}_{0}$ indicando uma maior representatividade da variabilidade espacial destes atributos na área de estudo.

A variação da razão de dependência (RD) foi de 3 a 50 indicando que os atributos apresentam um máximo de 50\% de aleatoriedade nos dados de amostragem. Todos os atributos apresentaram dependência espacial. Segundo Cambardella et al. (1994) a RD foi classificada como forte e moderada. Lima et al. (2010) afirmam que quanto maior a dependência espacial da variável melhor estrutura espacial e maior precisão podem ser obtidas na estimativa em locais não amostrados.

Tabela 2. Modelos e parâmetros estimados dos semivariogramas experimentais em área dos atributos do solo em diferentes camadas.

\begin{tabular}{|c|c|c|c|c|c|c|c|}
\hline \multirow[t]{2}{*}{ Parâmetros } & DMP & Ds & Est C & COT & Macro & Micro & VTP \\
\hline & \multicolumn{7}{|c|}{$0-0,05 \mathrm{~m}$} \\
\hline Modelo & Esf. & Esf. & Exp. & Exp. & Exp. & Exp. & Esf. \\
\hline $\mathrm{C}_{0}$ & 0,0035 & 0,0003 & 5,4000 & 6,4100 & 0,9100 & 0,9000 & 0,30 \\
\hline $\mathrm{C}_{0+} \mathrm{C}_{1}$ & 0,0073 & 0,0123 & 48.84 & 20,5900 & 8,5410 & 7,7080 & 8,11 \\
\hline${ }^{1} \mathrm{RD}$ & 49 & 3 & 11 & 31 & 11 & 12 & 11 \\
\hline $\mathrm{a}(\mathrm{m})$ & 53 & 14 & 17 & 28 & 19 & 15 & 13 \\
\hline$r^{2}$ & 0,85 & 0,92 & 0,94 & 0,80 & 0,88 & 0,52 & 0,70 \\
\hline \multirow[t]{2}{*}{${ }^{3} \mathrm{VC}$} & 0,89 & 0,82 & 0,78 & 0,83 & 0,70 & 0,76 & 0,96 \\
\hline & \multicolumn{7}{|c|}{$0,05-0,10 \mathrm{~m}$} \\
\hline Modelo & Esf. & Exp. & Exp. & Exp. & Exp. & Exp. & Exp. \\
\hline $\mathrm{C}_{0}$ & 0,0013 & 0,00127 & 4.10 & 0,4600 & 1,2400 & 1,2100 & 1,46 \\
\hline $\mathrm{C}_{0+} \mathrm{C}_{1}$ & 0,0275 & 0,0099 & 36.57 & 4,4690 & 11,7900 & 6,0080 & 12,07 \\
\hline${ }^{1} \mathrm{RD}$ & 5 & 13 & 11 & 10 & 11 & 20 & 12 \\
\hline $\mathrm{a}(\mathrm{m})$ & 21 & 24 & 15 & 17 & 19 & 34 & 17 \\
\hline$r^{2}$ & 0,90 & 0,97 & 0,91 & 0,74 & 0,90 & 0,61 & 0,84 \\
\hline \multirow[t]{2}{*}{${ }^{2} \mathrm{VC}$} & 0,69 & 0,88 & 0,72 & 0,82 & 0,67 & 1,01 & 0,65 \\
\hline & \multicolumn{7}{|c|}{$0,10-0,20 \mathrm{~m}$} \\
\hline Modelo & Exp. & Exp. & Esf. & Esf. & Exp. & Exp. & Exp. \\
\hline $\mathrm{C}_{0}$ & 0,0040 & 0,0011 & 5,70 & 2,251 & 1,0500 & 0,5200 & 5,7700 \\
\hline $\mathrm{C}_{0+} \mathrm{C}_{1}$ & 0,0354 & 0,0088 & 130,3 & 4,5030 & 9,5500 & 4,2670 & 11,550 \\
\hline${ }^{1} \mathrm{RD}$ & 11 & 12 & 4 & 50 & 11 & 12 & 50 \\
\hline $\mathrm{a}(\mathrm{m})$ & 17 & 21 & 14 & 43 & 19 & 19 & 53 \\
\hline$r^{2}$ & 0,69 & 0,85 & 0,75 & 0,91 & 0,88 & 0,94 & 0,94 \\
\hline${ }^{2} \mathrm{VC}$ & 0,99 & 0,98 & 0,95 & 0,81 & 0,61 & 0,80 & 0,72 \\
\hline
\end{tabular}

DMP: diâmetro médio ponderado; Ds: Densidade do solo; COT: carbono orgânico total; Esf.: Esférico; Exp.: Exponencial; $\mathrm{C}_{0}$ : efeito pepita; $\mathrm{C}_{0+} \mathrm{C}_{1}$ : patamar; ${ }^{1} \mathrm{RD}$ : razão de dependência espacial; $\mathrm{r}^{2}$ : coeficiente de determinação; ${ }^{2} \mathrm{VC}$ : validação cruzada. 
Os atributos DMP, Ds, Est C, COT, Macro, Micro e VTP apresentaram estruturas de dependência espacial conforme demonstrado pelos ajustes ao modelo esférico e exponencial. De maneira geral os atributos que mostraram maior e menor continuidade espacial foi o DMP e Ds na camada superficial. O DMP se ajustou ao modelo esférico com um raio de dependência espacial de 53m (alcance) e um RD de 49\% de aleatoriedade nos dados; para a Ds, o modelo foi o esférico com um raio de dependência espacial de $14 \mathrm{~m}$ e uma razão de aleatoriedade de $3 \%$.

Observando os mapas de krigagem para o COT e DMP (Figura 2) pode-se afirmar que há relação espacial, visto que as escalas de solo que apresentam os maiores valores do DMP nas camadas de 0-0,05 e 0,05-0,10m coincidem onde há predominância dos maiores valores do COT. O que enfatiza a importância do COT para a agregação do solo (Rozane et al., 2010). Em estudo realizado em diferentes manejos, Wendling et al. (2012) observou que o COT em área de pastagem apresentou maior teor de carbono quando comparado com outros manejos. Para Dos Santos et al. (2013) os elevados teores de COT são características de TPAs. É válido ressaltar que a agregação do solo pode ser alterada de forma direta pelo manejo e indireta por fatores bióticos e abióticos, sendo considerado como indicador físico da qualidade dos solos.

Observa-se que os menores valores de Est $C$ ocorreram nas camadas 0-0,05 e 0,05-0,10m, havendo um acréscimo na camada de 0,20-0,30m influenciado pelos maiores valores de Ds. Provavelmente os maiores valores de Ds na camada superficial são atribuídos ao pisoteio animal, uma vez que é notória a sua influência nas áreas periféricas, ou seja, nas bordas do mapa. Em estudos realizado por Alho et al. (2014), com agregados e estoque de carbono em campo natural e floresta, relataram que o conteúdo de COT foi determinante para os resultados de Est C.
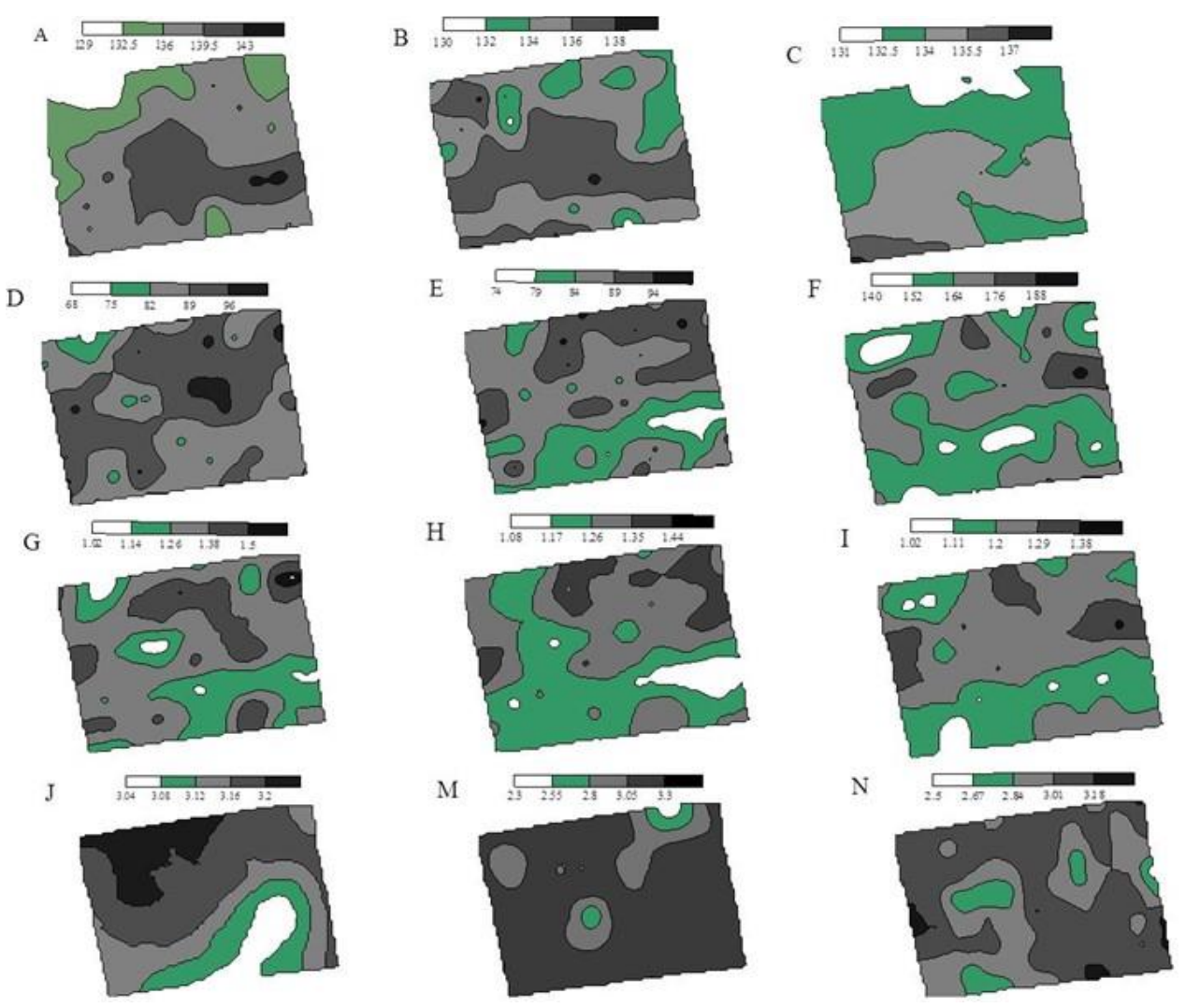

Figura 2. Mapas de distribuição espacial nas camadas 0-0,05, 0,05-0,10 e 0,10$0,20 \mathrm{~m}$, respectivamente para o carbono orgânico total (COT: A, B e C); estoque de carbono (Est C.: D, E e F); densidade do solo (Ds: G, H e I); diâmetro médio ponderado (DMP: J, M e N). 
O VTP apresentou correlação positiva com a Macro, o qual é verificado nos mapas destes atributos (Figura 3). A Macro é um atributo inteiramente ligado ao crescimento das plantas e seus valores ficaram acima de $0,10 \mathrm{~m}^{3} \mathrm{~m}^{-3}$. Segundo Kiehl (1979), valores abaixo de $0,10 \mathrm{~m}^{3} \mathrm{~m}^{-3}$ prejudicam o desenvolvimento das raízes, a infiltração de água e aeração do solo. Observa-se que os maiores valores da Micro se concentram na camada superficial $\left(0,32 \mathrm{~m}^{3} \mathrm{~m}^{-3}\right)$ indicando uma forte relação com a Ds (Figura 2). Já o VTP, apresentou seus valores quase contínuos nas primeiras camadas com acréscimo considerável na última camada (Figuras 4 e 5).

A

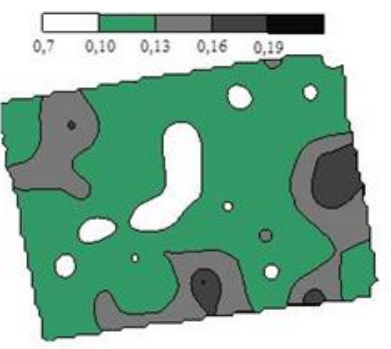

D

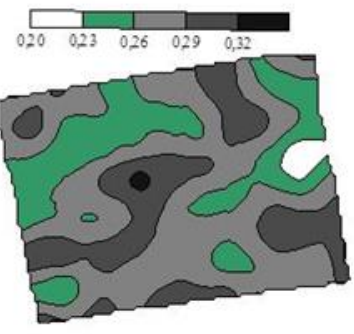

G

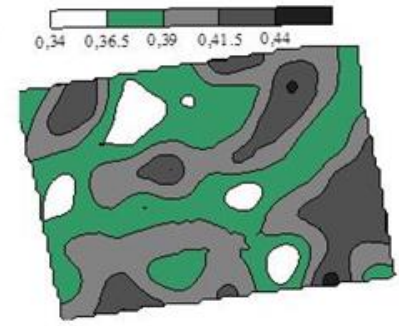

B

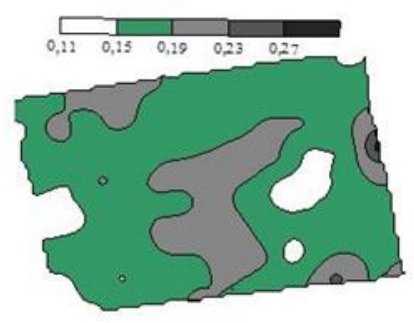

E

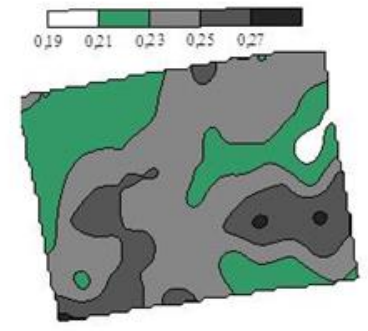

$\mathrm{H}$

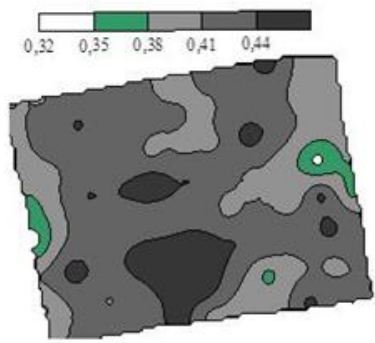

C

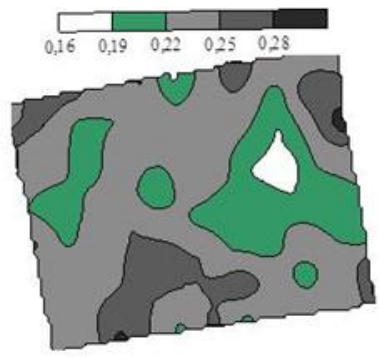

F

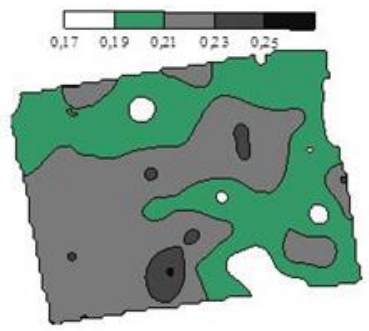

I

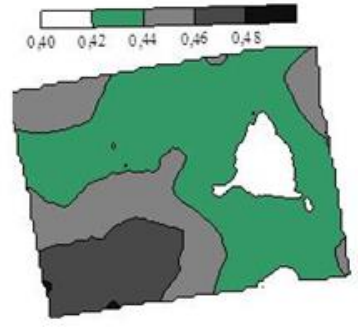

Figura 3. Mapas de distribuição espacial nas camadas $0-0,05,0,05-0,10$ e 0,10-0,20 m, respectivamente para a macroporosidade (Macro: A, B e C), microporosidade (Micro: D, E e F) e volume total de poros (VTP: G, H e I). 
A

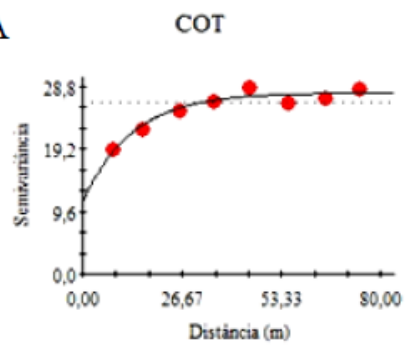

D

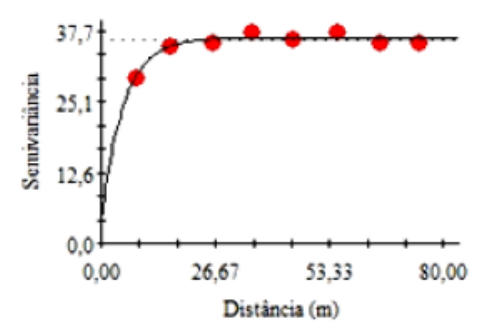

G

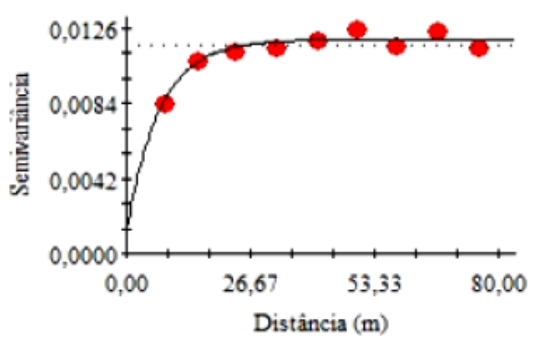

B

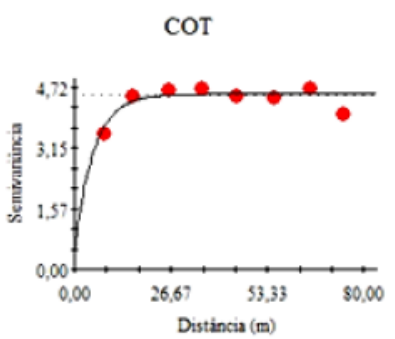

E

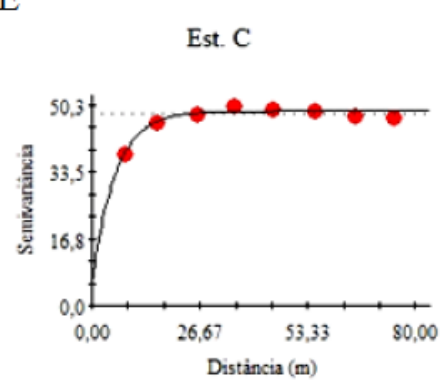

$\mathrm{H}$

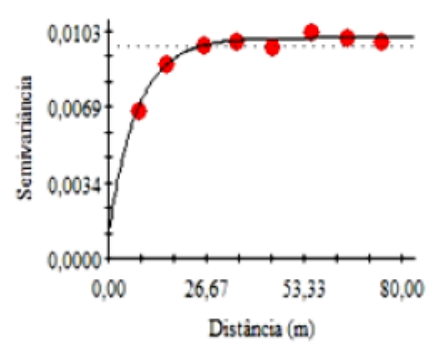

C COT

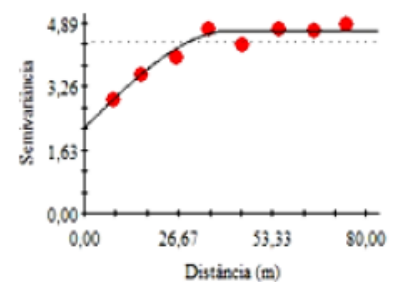

F

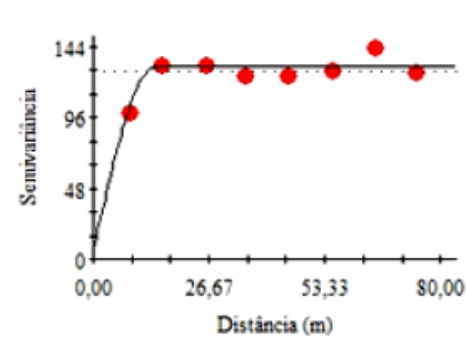

I

Ds

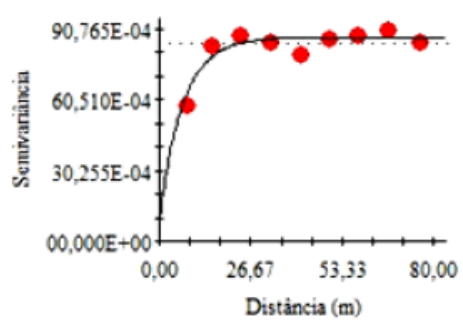

Figura 4. Semivariogramas experimentais nas camadas $0-0,05,0,05-0,10$ e $0,10-0,20 \mathrm{~m}$, respectivamente para o Carbono Orgânico Total (COT: A, B e C), Estoque de Carbono (Est. C: D, E e F) e densidade do solo (Ds: G, H e I).

\section{CONCLUSÕES}

De modo geral, mesmo estando inserida em área de pastagem, as variáveis Ds, Micro, Macro e VPT estiveram dentro dos valores de referências, os quais poderiam vir a restringir o desenvolvimento das plantas e a infiltração de água no solo.

O DMP e a Ds mostraram dependência de COT, pois os valores de ambos atributos aumentam ou diminuem de acordo com a camada.

Em camadas, os estoques de carbono estiveram mais relacionados à densidade do solo do que propriamente aos teores de carbono orgânico. 

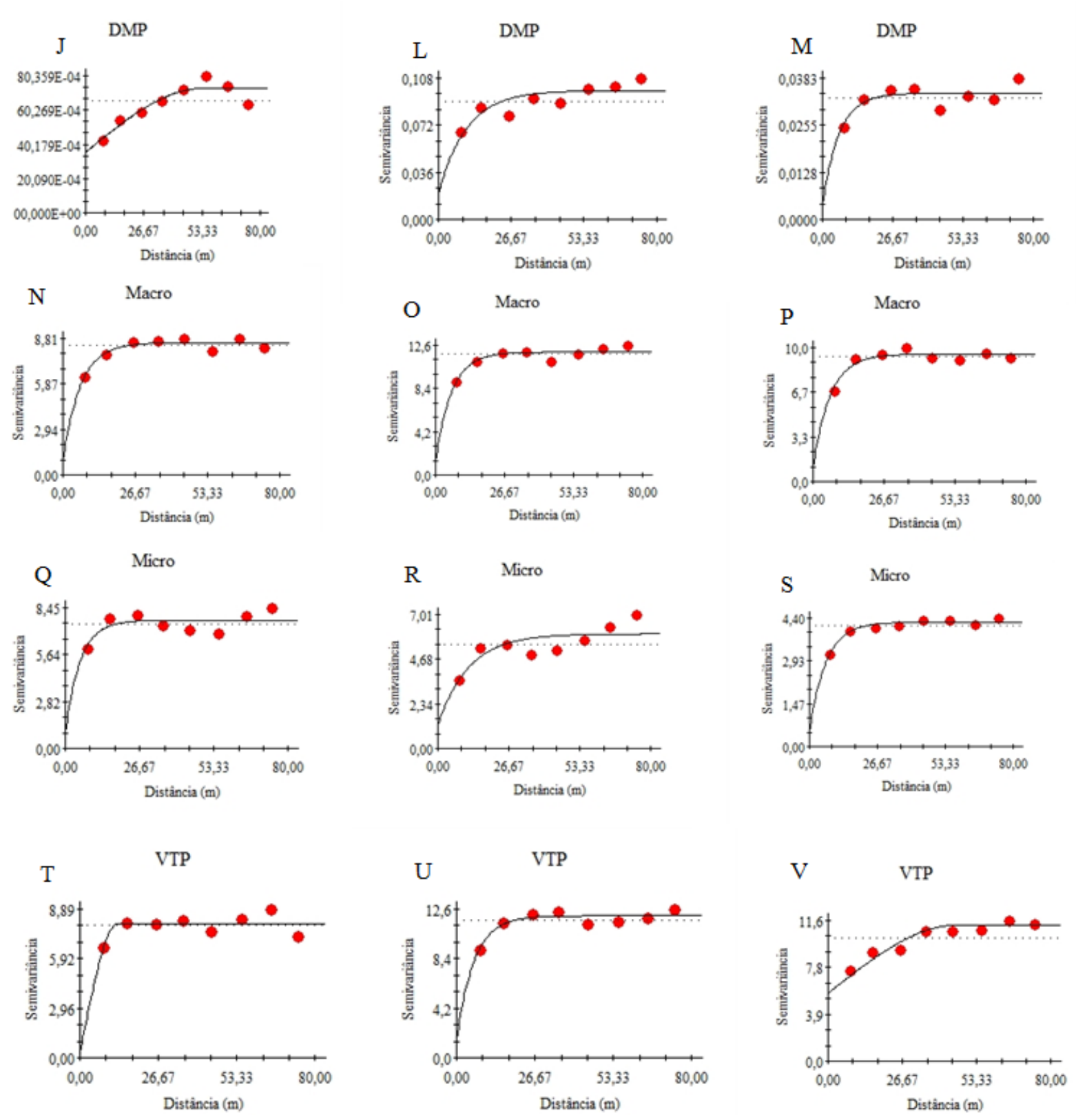

Figura 5. Semivariogramas experimentais nas camadas $0-0,05,0,05-0,10$ e $0,10-0,20 \mathrm{~m}$, respectivamente para o diâmetro médio ponderado (DMP: J, L e M), macroporosidade (Macro: N, O e P), microporosidade (Micro: G, H e I) e volume total de poros (VTP: T, U e V).

\section{REFERÊNCIAS}

ALHO, L. C.; CAMPOS, M. C. C.; SILVA, D. M. P.; MANTOVANELLI, B. C.; SOUZA, Z. M. Variabilidade espacial da estabilidade de agregados e estoque de carbono em Cambissolo e Argissolo. Pesquisa Agropecuária Tropical, v. 44, p. 246-254, 2014.

AQUINO, R. E.; CAMPOS, M. C. C.; MARQUES JÚNIOR, J.; OLIVEIRA, I. A.; MANTOVANELLI, B. C.; SOARES, M. D. R. Geoestatística na avaliação dos atributos físicos em latossolo sob floresta nativa e pastagem na Região de Manicoré, Amazonas.

Revista Brasileira Ciência do Solo, v. 38, p. 397-406, 2014b. http://dx.doi.org/10.1590/S0100-06832014000200004 
AQUINO, R. E.; CAMPOS, M. C. C.; OLIVEIRA, I. A. MARQUES JÚNIOR, J.; SILVA, D. M. P.; SILVA, D. A. Variabilidade espacial de atributos físicos de solos antropogênico e não antropogênico na região de Manicoré, Am. Bioscience Journal, v. 30, p. 988-997, 2014a. http://www.seer.ufu.br/index.php/biosciencejournal/article/view/21833

BRASIL. Ministério das Minas e Energia. Projeto Radambrasil, folha SB. 20, Purus. Rio de Janeiro, 1978. 561p.

BLAISKI, E.; TORMENA, C. A.; FIDALSKI, J.; GUIMARÃES, R. M. Quantificação de degradação física do solo por meio da curva de resistência do solo à penetração. Revista Brasileira Ciência do Solo, v. 32, p. 975-983, 2008. https://doi.org/10.1590/S010006832008000300007

CAMBARDELLA, C. A.; MOORMAN, T. B.; NOVAK, J.M.; PARKIN, T. B.; KARLEN, D. L.; TURCO, R. F.; KONOPKA, A. E. Field-scale variability of soil properties in central lowa soils. Soil Science of Society of America Journal, v. 58, p. 1501-1511, 1994. 10.2136/sssaj1994.03615995005800050033x

CAMPOS, M. C. C.; RIBEIRO, M. R.; SOUZA JÚNIOR, M. S.; RIBEIRO FILHO, M. R.; SOUZA, R. V. C. C.; ALMEIDA, M. C. Caracterização e classificação de terras pretas arqueológicas na Região do Médio Rio Madeira. Bragantia, v. 70, n. 3, p. 598-609, 2011.

CAMPOS, M. C. C. C.; SANTOS, L. A. C.; SIlvA, D. M. P.; MANTOVANELli, B. C.; SOARES, M. D. R. Caracterização física e química de terras pretas arqueológicas e de solos não antropogênicos na região de Manicoré, Amazonas. Revista Agro@mbiente On-line, v. 6, p. 102-109, 2012. http://dx.doi.org/10.18227/1982-8470ragro.v6i2.682

CAMPOS, M. C. C.; SOARES, M. D. R.; SANTOS, L. A. C.; OLIVEIRA, I. A.; AQUINO, E. A. Spatial variability of physical attributes in Alfissol under agroforestry, Humaitá region, Amazonas state, Brazil. Revista de Ciências Agrárias, v. 56, p. 149-159, 2013. http://dx.doi.org/10.4322/rca.2013.023

COUTINHO, F. S.; PEREIRA, M. G.; MENEZES, C. E. G.; GUARESCHI, R. F.; ASSUNÇÃO, S. A. Attributes of soil under agriculture, grazing and three succession stages of forest. Floresta e Ambiente, v. 24, p. 3-11, 2017. http://dx.doi.org/10.1590/2179-8087.091914

CENTRO DE PESQUISA DE RECURSOS MINERAIS - CPRM. Hidroclimatologia, geologia, recursos minerais, geomorfologia e unidades de paisagens. Manaus, 2001. 93p. (Relatório Técnico).

Dos SANTOS, L. A. C.; CAMPOS, M. C. C., AQUINO, R. E.; BERGAMIN, A. C.; SILVA, D. M. P.; MARQUES JUNIOR, J. et al. Caracterização de terras pretas arqueológicas no Sul do Estado do Amazonas. Revista Brasileira de Ciência do Solo, v. 37, p. 825-836, 2013. http://dx.doi.org/10.1590/S0100-06832013000400001

EMPRESA BRASILEIRA DE PESQUISA AGROPECUÁRIA - EMBRAPA. Manual de métodos de análise de solo. 2. ed. Rio de Janeiro: CNPS, 1997. 212p.

EMPRESA BRASILEIRA DE PESQUISA AGROPECUÁRIA - EMBRAPA. Centro Nacional de Pesquisa de Solos. Sistema Brasileiro de Classificação de Solos. Brasília, 353p. 2013. 
KÄMPF, N.; KERN, D. C. O solo como registro da ocupação humana pré-histórica na Amazônia. In: TORRADOVIDAL, P.; ALLEONI, L. R. F.; COOPER, M.; SILVA, A. P. (eds). Tópicos em ciência do solo. Viçosa: Sociedade Brasileira de Ciência do Solo, 2005. p. 277-320.

KEMPER, W. D.; CHEPIL, W. S. Size distribution of aggregates. In: BLACK, C. A. (eEd.). Methods of soil analysis. Madison: American Society of Agronomy, 1965. p.499-510.

KIEHL, E. J. Manual de edafologia: relações solo-planta. 1. ed. São Paulo: Agronômica Ceres, 1979. 264p.

LIMA, J. S. S.; SOUZA, G. S.; SILVA, S. A. Amostragem e variabilidade espacial de atributos químicos do solo em área de vegetação natural em regeneração. Revista Árvore, v. 34, p. 127-136, 2010. http://dx.doi.org/10.1590/S0100-67622010000100014

OLIVEIRA, I. A.; CAMPOS, M. C. C.; SOARES, M. D. R.; AQUINO, R. E.; MARQUES JÚNIOR, J.; NASCIMENTO, E. P. Variabilidade espacial de atributos físicos em um cambissolo háplico, sob diferentes usos na região sul do Amazonas. Revista Brasileira de Ciência do Solo, v. 37, p.1103-1112, 2013. http://dx.doi.org/10.1590/S010006832013000400027

ROCHA SILVA, F. W.; LIMA, H. N.; TEIXEIRA, W. G.; MOTTA, M. B.; SANTANA, R. M. Caracterização química e mineralogia de solos antrópicos (Terras Pretas de Índio) na Amazônia Central. Revista Brasileira de Ciência do Solo, v. 35, p. 673-681, 2011. https://doi.org/10.1590/S0100-06832011000300002

ROZANE, D. E.; CENTURION, J. F.; ROMUALDO, L. M.; TANIGUCHI, C. A. K.; TRABUCO, M.; ALVES, A. U. Estoque de carbono e estabilidade de agregados em um Latossolo Vermelho distrófico, sob diferentes manejos. Bioscience Journal, v. 26, p. 24$32,2010$.

SANTOS, D.; SOUZA, E. G.; NÓBREGA, L. H. P.; BAZZI, C. L.; GONÇALVES JÚNIOR, A. C. Variabilidade espacial de atributos físicos de um Latossolo vermelho após cultivo de soja. Revista Brasileira Engenharia Agrícola e Ambiental, v.16, p.843-848, 2012.

SOUZA, Z. M.; MARQUES JÚNIOR, J.; PEREIRA, G. T. Variabilidade espacial de atributos físicos do solo em diferentes formas do relevo sob cultivo de cana-de-açúcar. Revista Brasileira de Ciência do Solo, v. 28, p. 937-944, 2004.

STEINBEISS, S.; GLEIXNER, G.; ANTONIETTI, M. Effect of biochar amendment on soil carbon balance and soil microbial activity. Soil Biology and Biochemistry, v. 41, p.1301-1310, 2009. https://doi.org/10.1016/j.soilbio.2009.03.016

VASCONCELOS, R. F. B.; CANTALICE, J. R. B.; OLIVEIRA, V. S.; COSTA, Y. D. J.; CAVALCANTE, D. M. Estabilidade de agregados de um Latossolo Amarelo distrocoeso de tabuleiro costeiro sob diferentes aportes de resíduos orgânicos da cana-de-açúcar. Revista Brasileira de Ciência do Solo, v. 34, p. 309-316, 2010.

VELDKAMP, E. Organic carbon turnover in three tropical soils under pasture after deforestation. Soil Science Society American Journal, v. 58, p. 175-180, 1994. http://dx.doi.org/10.2136/sssaj1994.03615995005800010025x 
VIEIRA, S. R.; DECHEN, S. C. F.; SIQUEIRA, G. M.; DUFRAC, G. Variabilidade espacial de atributos físicos e químicos relacionados com o estado de agregação de dois Latossolos cultivados no sistema de semeadura direta. Bragantia, v. 70, p. 185-195, 2011.

WARRICK, A. W.; NIELSEN, D. R. Spatial variability of soil physical properties in the field. In: HILLEL, D. Applications of soil physics. New York: Academic Press, 1980. p. 319344.

WENDLING, B.; VINHA-FREITAS, I. C.; OLIVEIRA, R. C. de; BABATA, M. M.; BORGES, E. N. Densidade, agregação e porosidade do solo em áreas de conversão do cerrado em floresta de pinus, pastagem e plantio direto. Bioscence Journal, v. 28, p.256$265,2012$.

YEOMANS, J. C.; BREMNER, J. M. A rapid and precise method for routine determination of organic carbon in soil. Communication in Soil Science and Plant Analysis, v. 19, p. 1467-1476. 1988. https://doi.org/10.1080/00103628809368027 